directly into the catchpot, which in turn discharges into a vertical drain stack. The vertical acid-drain stacks occur at $10-\mathrm{ft}$. intervals, that is, at every steel stanchion between windows and on the centre lines of laboratory benches.

Service pipes to these benches branch off the lines under the window benches, the water, compressedair pipes and electricity conduits running under the reagent shelf spaced at $15 \mathrm{in}$. above bench-level, while the gas tubing is taken alongside the waste trough under the bench top. Only gas controls are on the bench top, and these are raised above benchlevel to reduce corrosion and to ease cleaning and polishing of the benches. Removal waste bins are an integral part of the bench design.

Polythene ('Vulcathene') has been used for lining the central waste troughs in the benches and the waste pipes from benches, drip sockets and sink traps in all laboratories. The latter, being precision made and thus identical, are readily interchangeable and saved much time and labour in the construction and erection of the benches. The bench tops are constructed of seasoned African teak, and alternate laboratory benches are fitted with a glazed sink or a readily removable inset so that a standard thermostat projecting 9 in. above the bench-level can be inserted. Water, gas and general fittings to the bench are of gun metal.

In all teaching and research laboratories, foul air is extracted at both floor- and ceiling-levels.

Fume cupboards in the teaching laboratories are in a special fume room at one end of the laboratory. The controls for the service points are external and mounted on a black plastic panel under the cupboard working surface. Each fume cupboard is lighted by fluorescent tube fixed above the working surface, and protected from fumes by a removable sheet of obscured glass. Fumes are extracted from the cupboards through high- and low-level outlets in the rear walls by exhaust motors situated in the roof of the building and individually controlled.

The research laboratories have not been individ. ually designed for specialized work, the services and layout being sufficiently flexible for the laboratory to be adapted to any type of chemical research work. Only a few of the laboratories have fixed benches, and in general the normal services have been made available from a 9-in. shelf attached to the wall at $3 \mathrm{ft}$. above the floor. This arrangement permits full use of space. Soft-wood tables (or metal frames where necessary) of various heights and designs are used for the erection of apparatus. Additional equip. ment in research rooms are wall channelling, and floor-and ceiling-tapped sockets which may be used for the attachment of blackboard, shelves, electrical apparatus and small-diameter 'scaffolding' for supporting a metal framework. The wall channelling is of a $\mathrm{C}$ cross-section, so that threaded bolts can be slid along to any position horizontally, the head of the bolt being in the channel and the threaded shank projecting outwards. The floor sockets are directly below those in the ceiling, and all are at 3-ft. centres, in both directions.

It is proposed to complete the present building within the next two years by the addition of a lecture theatre block comprising three lecture theatres seating 300,150 and 100 , respectively. This block will also house the library and small seminar rooms, and will form a third wing to the main spine. The spine will also be extended to accommodate the newly created Sub-department of Radiochemistry.

\section{HARWELL MEETING OF THE PHYSICAL SOCIETY}

THE spring meeting of the Physical Society was held at the Atomic Energy Research Establishment, Harwell, during March 31-April 2; the first day was taken up by discussion of topics connected with nuclear energy-levels, the second with topics connected with neutron physics, and there was a visit to the Harwell laboratories on the morning of April 2. About two hundred and fifty people attended, including several visitors from the Continent of Europe; most of the talks were given by physicists from Harwell and Oxford, the other contributions being from Cambridge and the Imperial College of Science and Technology, London.

The subject of nuclear energy-levels was introduced by B. H. Flowers (Harwell) with a review of the theory of the low-lying levels of nuclei. He directed attention to the contrast between the independent particle (the 'quasi-atomic') model of the nucleus, the chief success of which lies in light nuclei; and the collective, or 'quasi-molecular' model, which has accounted for many of the properties of heavy nuclei. In the first model, one takes a central core with one or more external particles, and considers the interactions between the particles and between the particles and the core. The interaction is usually taken, at one extreme, as pure spin-orbit ( $j-j$ coupling) or, at the other extreme, as a force derived from a central two-body potential ( $L-S$ coupling). Alternatively, a mixture of the two interactions (intermediate coupling) has been used with success, as Lane has shown with reference to the lithium isotopes and the mirror nuclei carbon-13 and nitrogen-13. Dr. Flowers suggested that experimental evidence should be sought for the odd-parity levels of nuclei between oxygen and calcium; near oxygen these levels probably correspond to $1 p$ particles being raised to the $2 s$ or $1 d$ shells, and near calcium to $1 d$ or $2 s$ particles being raised to the $2 p$ or $1 f$ shells. $\mathrm{He}$ said that the sequence of spins of these odd-parity levels in oxygen-17 has been observed, by comparison with fluorine-17, to be $1 / 2,7 / 2$ and $3 / 2$, as expected; and that he would expect the corresponding sequence of spins for calcium to be $7 / 2,3 / 2$ and $1 / 2$. For aluminium-25, intermediate between oxygen and calcium, he would expect an intermediate order, as has been found to be the case. Further theoretical evidence for the validity of intermediate coupling in nuclei with atomic masses between 18 and 20 was later presented by J. P. Elliott (Harwell); many of the experimental data can be fitted with a single value of the coupling parameter, and, in particular for fluorine-19, the energies of the lower even-parity levels, the probability of transitions between these levels, and the $\log f t$ values for oxygen-19 and neon-19 decay, can all be accounted for. Experimental evidence for the transition probabilities from the first four levels in fluorine-19, as determined by the gammarays from fast-neutron inelastic scattering, was presented by J. M. Freeman (Harwell) : the levels were established both by the gamma-ray energies and from excitation functions. In addition to wellestablished levels, there was good evidence for a level at $\mathbf{1} \cdot 43 \mathrm{MeV}$., probably of negative parity. G. Dearnaley (Cambridge) showed results of interesting interference effects which occur when protons or alpha-particles are scattered by fluorine nuclei : the 
interference, at a resonance where there is no spin change, occurs because of the interference between the compound nucleus and Rutherford scattering.

Proceeding to the collective or 'quasi-molecular' model of the nucleus, Dr. Flowers referred to the success of the Bohr-Mottelson picture of the rotational levels of even-even nuclei. The moment of inertia of the 'rotating' nucleus is $I=\gamma \beta^{2} I_{0}$, where $I_{0}$ is the moment of inertia of a rigid sphere, and $\beta^{2}$ is a distortion parameter. In the Bohr-Mottelson theory, which assumes no vortex motion, the coefficient $\gamma$ equals 1 : but since optical spectrometry (isotope shift) gives $\beta^{2}$, and nuclear levels give $\gamma \beta^{2}$, the coefficient $\gamma$ has been found experimentally to be $4 \pm 1$, in accordance with the theory of Blin-Stoyle and Weisskopf, which includes the orbital motions of the shell model. A study in the United States by Mrs. Scharff-Goldhaber has shown that if one plots $E_{2} / E_{1}$, the ratio of the experimentally determined energies of the second and first excited states of even-even nuclei, as a function of atomic weight, one finds, for heavy nuclei, the value of $3 \cdot 3$, as predicted from the Bohr-Mottelson theory. Near atomic mass 150, however, there is a sharp drop to a reasonably constant value $2 \cdot 1$, even though the individual values of $E_{1}$ vary between $0 \cdot 2$ and $2 \mathrm{MeV}$. Clearly, there is a collective feature present in the nuclei of intermediate weight. In the ensuing discussion, it appeared that the ratio of 2.5 for $E_{2} / E_{1}$ had been derived at Copenhagen by introducing two distortion parameters and large deviations from spherical symmetry. Experimental evidence on collective particle phenomena was given by J. O. Newton (Harwell) in a study of Coulomb excitation of odd $Z$-even $A$ heavy nuclei. In these, unless the projection of the angular momentum on the axis of deformation is $\frac{1}{2}$, the ratio $E_{2} / E_{1}$ should be a constant determined by the ground-state $J$ : the experimental results give satisfactory agreement for neptunium-237 and uranium233 (both spin 5/2) and for plutonium-239 (spin 1/2, and therefore no relation for $E_{2} / E_{1}$ ). For uranium235 , the data could fit spin $5 / 2$ or $7 / 2$, and bear no relation to the levels observed in the alpha decay of plutonium-239 ; this confirms that, in the latter, the levels excited are not the rotational levels based on a ground-state of uranium-235.

Part of one session was given to work from the Harwell cyclotron. Protons scattered out of the cyclotron at $20^{\circ}$ from carbon are 70 per cent polarized, and M. J. Brinkworth and B. Rose showed that by slowing down the protons in aluminium to the energy range 2-10 $\mathrm{MeV}$. and scattering a second time in helium, the sign of the polarization can be determined from the known scattering of protons in helium. The sign of the polarization so determined agrees with the sign predicted by Fermi on the basis of the shellmodel spin-orbit interaction. Total cross-sections and angular distributions for $140-\mathrm{MeV}$. protons scattered by beryllium, carbon, nitrogen and oxygen were discussed by A. E. Taylor and E. Wood. The angular distributions showed large Coulomb-nuclear interference and were in agreement with the modified optical model predictions; the total nuclear crosssections showed close agreement with neutron total cross-sections, except for beryllium which is an odd neutron nucleus. Measurements of total cross-sections with light and heavy water and with liquid hydrogen showed close agreement between $n-n$ (from the $n-D$ and $n-p$ difference) and $p-p$ scattering, and between the two values of $n-p$, direct and from the $p-D$ and $p-p$ difference. R. Wilson (Oxford) reported measure- ments of neutron inelastic cross-sections for carbon, aluminium, copper, cadmium and lead in the energy range 55-140 MeV. The results showed little variation with energy, in contrast to the total cross-sections. Measurements of the elastic scattering for the same elements and for lithium, beryllium, and nitrogen and oxygen molecules at $135 \mathrm{MeV}$. indicated that the $0^{\circ}$ scattering cross-sections were larger than those expected on the optical model, and that the potential in the optical model does not fall to zero at this energy.

The main subject dealt with in the field of slowneutron physies was the interaction of thermal neutrons with solids and liquids, the subject being introduced by R. J. Elliott (Harwell). Thermal neutrons have wave-lengths in the range 1-10 A. and are therefore useful for studying the structure of solids; and since the cross-sections of most nuclei are of the same order of magnitude, the positions of atoms such as hydrogen, which are difficult to find by X-rays, can be obtained with neutrons. There is also an interaction between the neutron's magnetic moment and the magnetic moment of an atom, so that magnetic order can be studied with neutrons as well.

The location of hydrogen atoms in organic compounds was later discussed in a paper by G. E. Bacon (Harwell). He said that once the problem of extinction had been properly understood, it was realized that large single crystals could be used for neutron diffraction studies, and this development has made the study of the position of hydrogen atoms in organic compounds feasible. At present, work is going on at Harwell and in the United States at Oak Ridge on several organic compounds. Because of the relatively weak neutron beams available from reactors at the present time, the substances under study are those for which some X-ray data are available: the technique is then to obtain, by Fourier analysis, a projection of the crystal on a single plane only. Bacon showed the results he has obtained on $\alpha$-resorcinol, in which the positions of the hydrogen atoms have been accurately established for the first time: in this work he used a crystal $1 \mathrm{~cm} . \times 2 \mathrm{~mm} . \times 3 \mathrm{~mm}$.

Continuing his review, Dr. Elliott explained that, in addition to the elastic scattering which is being studied in these experiments, the inelastic processes in which the neutron exchanges energy with the solid are of great interest. Since the energy of thermal neutrons is of the same order as the separation of the energy-levels of solids, the excitation or deexcitation of the levels can be studied comparatively easily. Again, because of the nuclear and magnetic interactions, the information obtained is related to both the vibrational and magnetic energy-states of the solid. Unfortunately, because of the relatively weak neutron beams available, really detailed experiments are not yet feasible. However, R. D. Lowde (Harwell) described the data he has obtained from a study of the inelastic scattering of a beam of thermal neutrons by a single erystal of iron. Here both the vibrational and magnetic type of inelastic scattering are important; but by suitable techniques the observational data may be separated into the two components. The magnetic scattering has been compared to that predicted by the Heisenberg and the collective-electron theories of ferromagnetism. At present, the data are accounted for satisfactorily by the Heisenberg model only. Further studies in the neighbourhood of the Curie point have confirmed 
that the magnetic scattering reaches a maximum there, and that that phenomenon is analogous to 'critical opalescence'.

The scattering of neutrons by crystalline solids can be described in a relatively exact fashion because the positions of the atoms are relatively well defined. However, Dr. Elliott continued, when more complex substances such as liquids are considered, the scattering is described in terms of a pair distribution function (that is, the distribution of atoms in the liquid as seen from an atom at the origin). For $\mathrm{X}$-ray scattering, only the dependence of the pair distribution function upon the distance between atoms is measured, but for neutron scattering the dependence upon time as well is important. The detailed behaviour of the pair distribution function with respect to time and distance can be found by measuring the differential scattering cross-section per unit solid angle and unit interval of the energy of the scattered neutron.

Again, detailed study of this kind is not yet feasible; but a preliminary experiment on the scattering of $14^{\circ} \mathrm{K}$. neutrons by solid and liquid lead was described by P. A. Egelstaff (Harwell). The object of the work was to compare the interaction of the neutrons with the thermal vibrations of the solid and liquid. It was shown that the chief features of the experimental data can be explained by assuming that the liquid is "a solid with the atoms placed at random positions". However, some features of the forward scattering require a more detailed model for their explanation.

Limitation of space does not permit a detailed review of all the contributions to the meeting, but mention should be made of the subject of very short half-lives. Prof. S. Devons described the work at the Imperial College of Science and Technology, London, on measurements of absolute gamma transition probabilities by the Doppler shift of the gamma-rays emitted by a recoiling compound nucleus. Here, unexpectedly, the chief unknown has proved to be the rate of slowing down of the heavy particles in the material into which they recoiled. Information is being sought from the recoil of ${ }^{7} \mathrm{Be}^{*}$ into varying thicknesses of copper: it was noted that the experimental values of the absolute transition probabilities for " $\mathrm{Be}$ * and ' $\mathrm{Li}$ * lie respectively above and below the values predicted by intermediate coupling theory. C. F. Coleman (Harwell) described a method of determining half-lives down to $10^{-11}$ sec. by means of fast coincidence techniques. The method depends on $\beta \gamma$ coincidences between two identical detectors placed at $180^{\circ}$ with respect to the sample, and differing only in the absorber screening the gamma detector. Alternating the absorber between the detectors enables small delays between the detectors to be cancelled out. The life-times observed (in units of $10^{-12}$ sec.) are : magnesium-24, $30 \pm 2$; titanium$46,7 \pm 4$; nickel-60, $8 \pm 5$; selenium-76, $25 \pm 10$; tellurium-122, $196 \pm 5$; and tellurium-124, $13 \pm 12$.

M. A. Grace (Oxford) described experiments on the alignment of cobalt-57 at very low temperatures. The determination of polarization correlation with aligned nuclei enabled him to determine the degree of $E_{2}$ and $M_{1}$ admixtures in the $123 \mathrm{keV}$. gamma-ray. R. Batchelor (Harwell) described a helium-3 spectrometer for use in the $100-1,000 \mathrm{keV}$. neutron range. The cross-section of helium-3 in the ${ }^{3} \mathrm{He}(n, p) \mathbf{T}$ reaction has given results in good agreement with those derived from detailed balancing, and the instrument has been used for determining the intens- ity and angular distribution near threshold of the ${ }^{7} \mathrm{Li}(p, n)^{7} \mathrm{Be}^{*}$ reaction. Of the $\mathrm{Cu}(n, p)$ reaction at $14 \mathrm{MeV}$. neutron energy, D. L. Allan (Harwell) showed that the number of protons below the conventional Coulomb barrier at $7 \mathrm{MeV}$. is far in excess (85 per cent of the total) of those expected, and bears a general resemblance to the distribution observed by Graves and Rosen of the neutrons from the parallel $\mathrm{Cu}(n, n)$ reaction. Finally, the redetermination of the slow-neutron capture cross-section of hydrogen was reported by C. H. Collie and R. E. Meads (Oxford). Neutrons from a pulsed D-D source were slowed down in a tank, and the gamma-rays from their capture were detected in a scintillation counter. The life-time, obtained from the decay-rate of the gamma-ray yield integrated over the tank, was $203 \pm 2 \mu$ sec., corresponding to a capture crosssection of 0.335 barn for a neutron velocity of $2,200 \mathrm{~m}$. $/ \mathrm{sec}$.

Other contributors were L. E. Beghian (Oxford) on gamma-rays from fast-neutron scattering; D. B. Gayther and K. P. Nicholson (Harwell) on neutron total cross-sections at intermediate energies; M. J. Poole (Harwell) on the spectrum of neutrons from a water moderator by time-of-flight analysis; P. C. Price (Cambridge) on the radiative capture of alphaparticles in nitrogen; E. R. Rae and J. E. Lynn (Harwell) on the analysis of levels in uranium-238 with a time-of-flight spectrometer ; T. H. R. Skyrme (Harwell) on the alpha-particle and shell models of the nucleus; and J. F. Turner (Harwell) on the lowintensity radiations in the decay of cobalt- 60 .

$$
\text { W. D. ALLen }
$$

\section{P. A. Egelstafi}

\section{MATERIALS FOR RADIO VALVES}

A $\mathrm{T}$ a meeting of the Radio Section of the A Institution of Electrical Engineers on March 21, a discussion was opened by Dr. R. O. Jenkins on the subject of "Materials for Valves". He stated that, historically, the technique of valve-making was derived from that of lamp-making, so that valves tended to use materials originally developed for lamps. With recent advances, however, modern valves require different and, in some cases, new materials; and since the quantities required are relatively small, it has not always been easy to persuade the supplier of materials to meet the very stringent specification of the valve manufacturer.

In addition to the opening speaker, nine others participated in the discussion. It was generally agreed that most of the materials used in valve manufacture are capable of great improvement, and it was suggested that the preparation of idealized specifications would encourage the supply of satisfactory materials in adequate quantities. The subject was surveyed under the three main headings of cathodes and heaters; other electrodes; and envelopes of both insulators and metals.

It was recognized that in Britain the use of cathode cores made of nickel with a low silicon content has avoided many of the difficulties due to interface troubles experienced in the United States and on the Continent of Europe, where a nickel of high silicon content has been used. The usual material in Britain is a pure commercial nickel with a low sulphur content and $0.05-0.10$ per cent of magnesium as a reducing agent. In more recently developed types of valves, however, even the low quantity of silicon 